\title{
Bench-to-bedside review: Functional hemodynamics during surgery - should it be used for all high-risk cases?
}

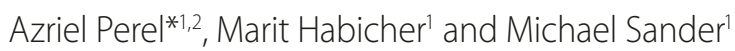

\begin{abstract}
The administration of a fluid bolus is done frequently in the perioperative period to increase the cardiac output. Yet fluid loading fails to increase the cardiac output in more than $50 \%$ of critically ill and surgical patients. The assessment of fluid responsiveness (the slope of the left ventricular function curve) prior to fluid administration may thus not only help in detecting patients in need of fluids but may also prevent unnecessary and harmful fluid overload. Unfortunately, commonly used hemodynamic parameters, including the cardiac output itself, are poor predictors of fluid responsiveness, which is best assessed by functional hemodynamic parameters. These dynamic parameters reflect the response of cardiac output to a preload-modifying maneuver (for example, a mechanical breath or passive leg-raising), thus providing information about fluid responsiveness without the actual administration of fluids. All dynamic parameters, which include the respiratory variations in systolic blood pressure, pulse pressure, stroke volume and plethysmographic waveform, have been repeatedly shown to be superior to commonly used static preload parameters in predicting the response to fluid loading. Within their respective limitations, functional hemodynamic parameters should be used to guide fluid therapy as part of or independently of goal-directed therapy strategies in the perioperative period.
\end{abstract}

\section{Current practice of perioperative fluid administration - more questions than answers} The importance of optimal fluid administration in the perioperative period is well recognized, since administering

\footnotetext{
*Correspondence: perelao@shani.net

${ }^{2}$ Department of Anesthesiology and Intensive Care, Sheba Medical Center, Tel Aviv University, Tel Hashomer, Tel Aviv 52621, Israel

Full list of author information is available at the end of the article
}

insufficient fluids or administering too much may both result in bad outcome. This challenge is further compounded by the fact that fluid requirements vary greatly among patients. One of the recommended strategies is that of fluid restriction, with the aim of preventing interstitial edema and postoperative weight gain that may lead to severe complications [1]. However, restrictive regimes of fluid management have been using fixed formulas based on body weight and have not, in general, individualized fluid administration according to the real needs of the patient.

Another prevalent strategy is that of goal-directed therapy (GDT), which has been repeatedly shown to significantly improve both short-term and long-term outcome $[2,3]$. The GDT approach is centered upon the maximization (optimization) of cardiac output $(\mathrm{CO})$ by incremental fluid administration. According to a commonly used protocol, fluid boluses should be repeatedly given as long as the resulting increase of the stroke volume (SV) exceeds $10 \%$ or when the SV decreases by more than 10\% [4]. However, a reduction in SV $>10 \%$ has been found to have very low sensitivity in justifying fluid administration and may partially account for the fact that GDT is usually associated with the administration of larger amounts of fluids compared with standard care [6,7]. The administration of large amounts of fluids is a potential source of worry since these fluids, especially crystalloids, do not stay in the intravascular space for a long period of time [8]. Moreover, a forgiving attitude towards aggressive fluid administration may encourage practitioners with inadequate training to overload their patients with fluid without appropriate monitoring.

\section{Inadequacy of commonly used parameters in guiding fluid administration}

The most common parameters being used to guide perioperative fluid management include clinical experience, urine output, mean arterial pressure and central venous pressure (CVP) $[9,10]$. Although these parameters are all important components of hemodynamic assessment, none of them has been shown to be a good predictor of 
the response of $\mathrm{CO}$ to fluid administration. This is true not only for the CVP [11,12], but also for more reliable preload parameters such as the left ventricular (LV) enddiastolic area and the global end-diastolic volume [13-15] (Figure 1). This inadequacy is further reflected in the fact that only one-half of critically ill and high-risk surgical patients, in whom fluid loading seems to be indicated, do indeed increase their $\mathrm{CO}$ in response to fluid loading (responders), while the other half (nonresponders) is loaded with fluids unnecessarily [16]. The inadequacy of commonly used hemodynamic parameters as predictors of the response to fluids stems from the fact that this response depends not only on the preload status, but also on the contractile state of the heart [17]. In making a decision about fluid administration it would therefore serve us best to rely on a measure of the slope of the LV function curve. This dynamic parameter is termed fluid responsiveness, and is a measure of the change in $\mathrm{CO}$ in response to a change in preload [18].

In clinical practice, fluid responsiveness is very often assessed by a fluid challenge. This common clinical approach, which constitutes the first step in most optimization protocols, has some inherent flaws. Detecting the expected increase in $\mathrm{CO}$ is not a trivial task, because relying on changes in blood pressure alone may lead to many false negative results [19]. More reliable methods for the accurate assessment of the effect of a fluid challenge include detection of a change in the CVP of at least $2 \mathrm{mmHg}$ [20] or, preferably, a change in the continuously measured CO itself [21]. However, relying on a fluid challenge to determine fluid responsiveness may eventually result in detrimental fluid overload in view of the large proportion of non-responders [16] and the need to determine fluid responsiveness repeatedly in some cases.

We hope the reader is by now convinced that having parameters which can predict fluid responsiveness accurately without the actual administration of fluids is of great clinical importance. These parameters, which we define in this context as functional hemodynamic parameters (FHP) or dynamic parameters, are able to reflect the response of the $\mathrm{CO}$ to a preload-modifying maneuver, to predict fluid responsiveness, and to obviate the need for time-consuming, potentially damaging, repeated fluid challenges.

\section{Functional hemodynamic parameters based on the} hemodynamic effects of a mechanical breath

To better understand this category of dynamic parameters, we need to briefly revise the physiological principles of heart-lung interaction during mechanical ventilation [22-25]. The main hemodynamic effect of the increase in intrathoracic pressure during a mechanical breath is a transient decrease in venous return. This decrease in venous return causes a decrease in the right ventricular stroke output, and, after a few beats, in the LV stroke output. It is important to note that the first and immediate effect of the rise in intrathoracic pressure is normally an augmentation of the LV SV, which is mainly due to the inspiratory squeezing of the pulmonary blood volume, an important reservoir of LV preload, and due to the decrease in the transmural aortic pressure that is equivalent to an effective decrease in $\mathrm{LV}$ afterload $[22,26,27]$. A mechanical breath will thus normally induce a cyclical biphasic change in LV ejection, which is composed of an early inspiratory augmentation followed by a decrease. This latter transient decrease in $\mathrm{CO}$ is a reflection of fluid responsiveness and becomes more pronounced in the presence of hypovolemia. These changes in the $\mathrm{CO}$ are reflected in the arterial pressure waveform, which may therefore provide readily measurable parameters of fluid responsiveness (Figures 2 and 3 ).

\section{Systolic pressure variation}

Systolic pressure variation (SPV) is the difference between the maximal and minimal values of systolic arterial pressure during one mechanical breath (Figure 3). The SPV is composed of an early inspiratory increase in the systolic blood pressure, termed delta up (dUp), which reflects the inspiratory augmentation of the $\mathrm{CO}$, and a later decrease in systolic blood pressure, termed delta down, which reflects the decreased $\mathrm{CO}$ due to the decrease in venous return (Figure 3) [18,28]. The SPV has been repeatedly shown, both experimentally and clinically, to accurately reflect fluid responsiveness, and to do so better than commonly used hemodynamic parameters $[13,14,28]$. The SPV has been validated and used to guide fluid therapy in a variety of surgical patients, including those undergoing major abdominal surgery [29], neurosurgical operations [30], vascular surgery [13], cardiac surgery [15] and scoliosis surgery [31,32].

Although the SPV has been found to be somewhat less accurate than pulse pressure variation (PPV) $[15,16,33]$, its accuracy in predicting fluid responsiveness is similar to that of stroke volume variation (SVV) $[15,16]$ (Figure 1). When PPV and SVV are not measured automatically, SPV has a distinct practical advantage over the other two parameters in that it can easily and accurately be estimated from visual examination of the arterial waveform tracing [34], while eyeballing the PPV is much less accurate [35].

\section{Pulse pressure variation}

PPV reflects the respiratory-induced changes in pulse pressure, and is calculated as the difference between the maximum and minimum pulse pressure values during one mechanical breath divided by their mean $[33,36]$ 

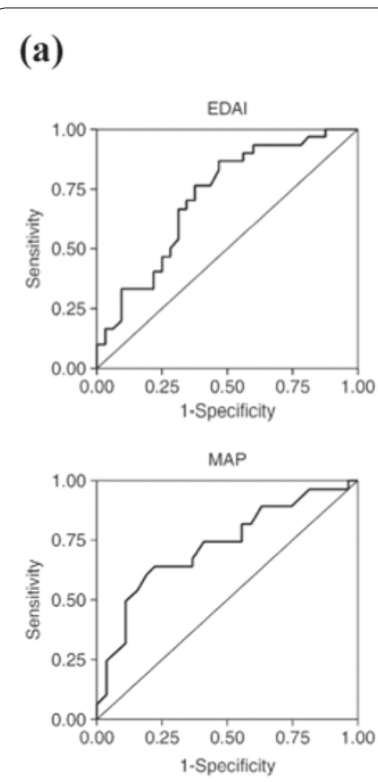
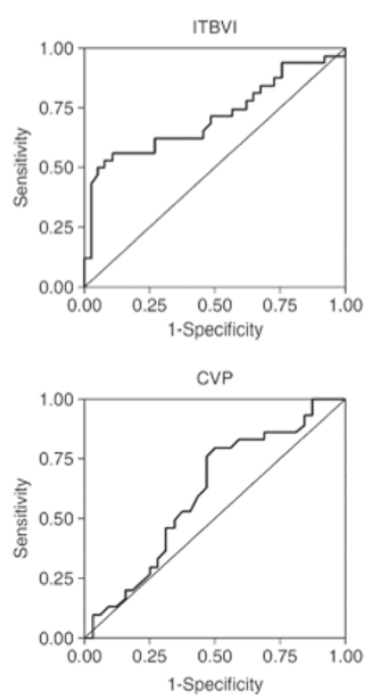

(b)
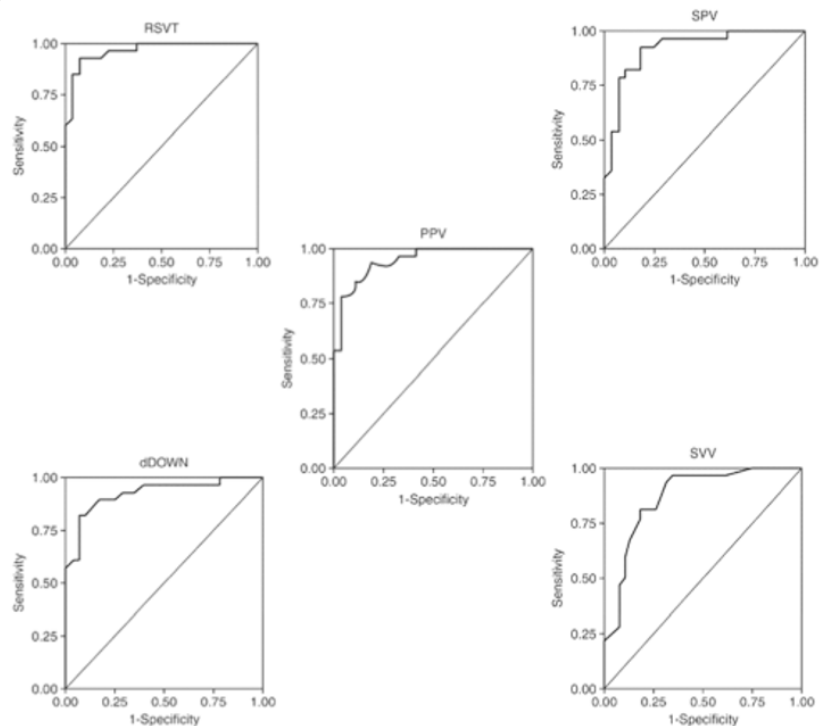

Figure 1. Static and dynamic parameters used to guide fluid management. (a) Receiver operating characteristic curves of static parameters: left ventricular end-diastolic area (EDAi), intra-thoracic blood volume index (ITBVI), mean arterial pressure (MAP) and central venous pressure (CVP). (b) Receiver operating characteristic curves for functional hemodynamic parameters: respiratory systolic variation test (RSVT), systolic pressure variation (SPV), pulse pressure variation (PPV), Delta down (dDown) and stroke volume variation (SVV). Note the obvious superiority of dynamic parameters over static parameters in predicting fluid responsiveness. Reproduced with permission from [15].

(Figure 3). PPV is somewhat more accurate than SPV since, under constant conditions, the SV is proportional to the pulse pressure, and since the changes in the systolic blood pressure may be influenced by some degree of transmission of airway pressure [16]. A less appreciated characteristic of PPV is that when hypovolemia develops and the filling of the aorta is greatly decreased, the changing relation of the SV to the pulse pressure causes the associated increase in PPV to be much greater than the simultaneous increase in SPV and SVV [37].

Numerous studies have repeatedly shown that PPV is an accurate predictor of fluid responsiveness with threshold values of 11 to $13 \%$ [17,25,38]. PPV has been validated and used to guide fluid therapy in a variety of surgical patients, which include those undergoing major abdominal procedures [39], liver transplantation [40], cardiac surgery [15,41-43] and scoliosis surgery [44]. Additionally, a high PPV value was associated with higher inflammatory response and lower organ yield in braindead organ donors [45]. According to a recent report, PPV in the range 9 to $13 \%$ could not reliably predict fluid responsiveness in $25 \%$ of anesthetized patients [46]. The possible reasons for this finding will be discussed later in Limitations of functional hemodynamic parameters. Automatic measurement of PPV is available in most monitors that use pulse contour analysis for the measurement of continuous $\mathrm{CO}$, and also in some standard monitors.

\section{Stroke volume variation}

SVV is the difference between the maximum and minimum SV during one mechanical breath divided by the mean SV (Figure 3). A threshold value of $10 \%$ was originally described in neurosurgical patients as differentiating responders from nonresponders [47]. Continuous measurement of this parameter has become possible with the introduction of pulse contour methods for the continuous measurement of CO. SVV has been validated and used to guide fluid therapy in a variety of surgical patients, including patients undergoing cardiac surgery [15,48-51], major abdominal surgery [52,53] and liver transplantation [54]. Although from a pure physiological viewpoint SVV should reflect best fluid responsiveness, the inherent limitations of pulse contour methods make SVV somewhat less accurate than PPV [16]. In addition, because of different nonstandardized proprietary algorithms, SVV values measured by one monitor cannot be used interchangeably with those measured by another monitor without great caution [55].

\section{Plethysmographic waveform variation}

Since pulse oximetry is a standard non-invasive intraoperative monitor, respiratory plethysmographic waveform variation (PWV) is the most commonly available dynamic parameter in mechanically ventilated anesthetized patients [56]. PWV can be calculated as the difference between the maximal and minimal plethysmographic 


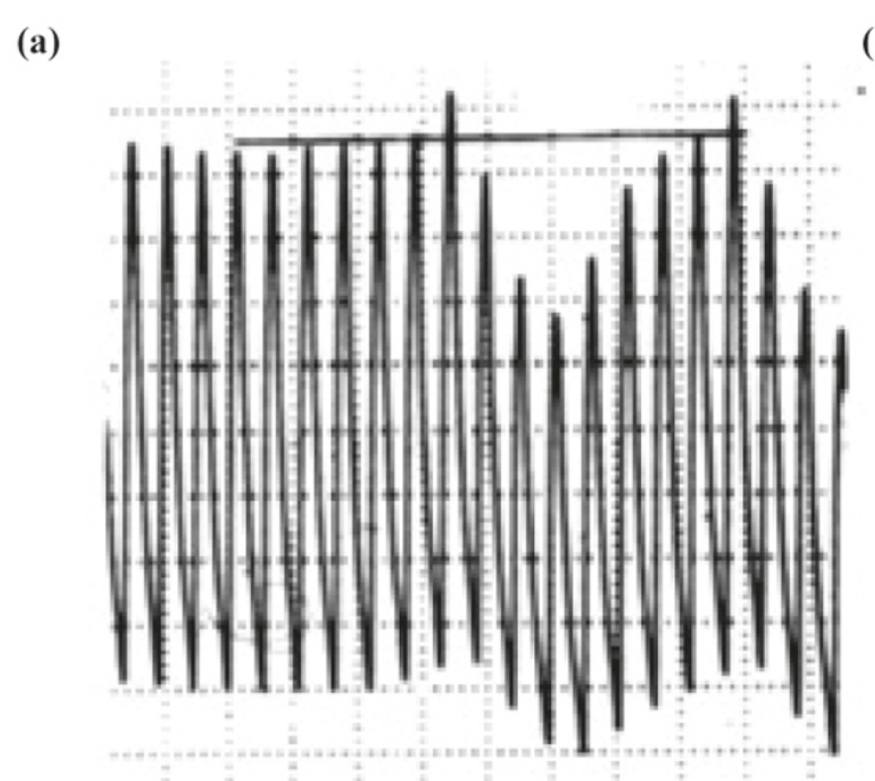

(b)

Figure 2. The arterial pressure waveform. (a) Responder and (b) nonresponder.

signal amplitudes divided by the amplitude of the signal during apnea or by the mean of the two values [57]. However, there are other variables and terms that are used to describe and measure PWV that may differ from each other [55]. In the absence of an automated measurement [58], the variations in the plethysmographic signal should be simply eyeballed, although there are no data regarding the sensitivity and accuracy of such observation. PWV has been shown to accurately reflect changes in circulating blood volume intraoperatively $[31,57]$ and in fluid responsiveness in patients undergoing major abdominal surgery $[5,59]$ and cardiac surgery $[58,60]$.

The major problem with the clinical use of PWV is the significant impact of vasoconstriction (for example, during hypotension or hypothermia) on the plethysmographic waveform. An increase in PWV may be the first sign for development of a still-occult hypovolemia and should prompt the anesthesiologist to consider the immediate administration of fluids.

\section{Other functional hemodynamic parameters}

A detailed description of the dynamic parameters that are based on echocardiographic assessment in critically ill patients is beyond the scope of this article. These parameters include the collapsibility index of the superior vena cava [26], and respiratory variations in the diameter of the inferior vena cava, in aortic blood flow velocity (also measured by esophageal Doppler) and in the aortic velocity-time integral.

A dynamic parameter of major interest is the response of the $\mathrm{CO}$ to passive leg-raising [61], a maneuver that can theoretically be used in the many anesthetized patients in whom pressure-derived FHP cannot be used [56]. In contrast to a mechanical breath that normally reduces $\mathrm{CO}$, passive leg-raising causes an endogenous fluid challenge that will increase $\mathrm{CO}$ in responders. The execution of passive leg-raising necessitates a major positional change, which makes it impractical intraoperatively. There are instances in the operating room, however, where postural changes may induce a hemodynamic response that might serve as a diagnostic maneuver of fluid responsiveness. Such response is best evaluated when a continuously measured $\mathrm{CO}$ is available and in the absence of pain or discomfort caused by the positional change itself.

A recently described dynamic parameter that may be of potential interest intraoperatively is the response to a 15-second end-expiratory occlusion, which may act like a fluid challenge due to the temporary absence of the cyclic impediment in venous return [62]. More recently, passive leg-raising and the end-expiratory occlusion were shown to perform better than PPV in patients with low respiratory system compliance [63], but their value in the operating room still needs to be defined.

\section{Limitations of functional hemodynamic parameters}

To properly measure and interpret FHP it is imperative that their many limitations be well recognized. The fact that any dynamic parameter is composed of a stimulus and a response [64] makes the process vulnerable to many confounding factors, which limit the number of anesthetized patients in which these parameters can be used [56]. 
(a)

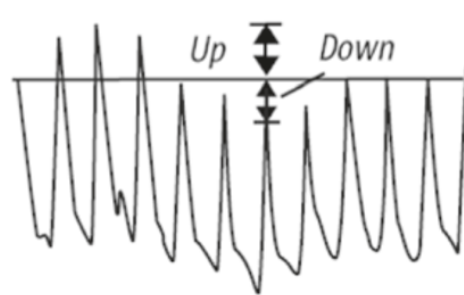

(b)

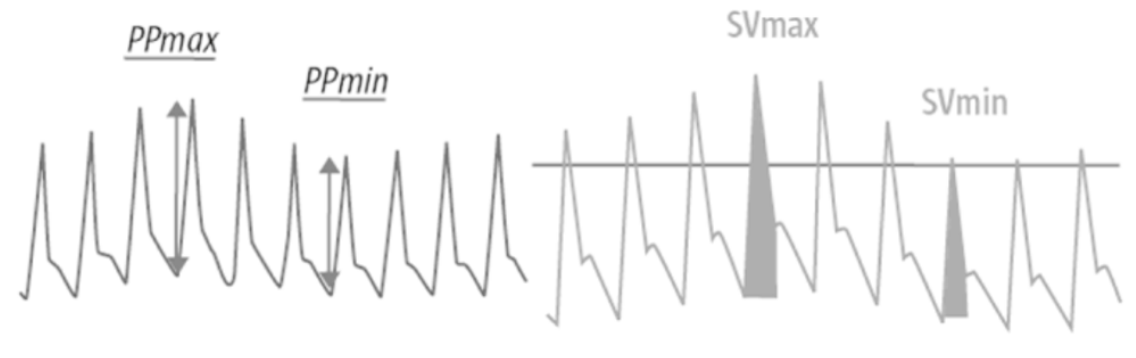

Figure 3. Principles for calculation of dynamic parameters based on the hemodynamic effects of a mechanical breath. (a) Systolic pressure variation. (b) Pulse pressure variation. PPmax, maximum pulse pressure; PPmin, minimum pulse pressure. (c) Stroke volume variation. SVmax, maximum stroke volume; SVmin, minimum stroke volume.

\section{Spontaneous breathing}

The hemodynamic effects of a spontaneous breath are physiologically different from those of a mechanical one and are greatly affected by the inspiratory effort. Dynamic parameters, especially when automatically measured, can therefore be very misleading in the presence of spontaneous or assisted ventilation. This is a major limitation of FHP and is responsible for the large number of anesthetized patients (for example, those under regional anesthesia) in whom these parameters cannot be used [56].

\section{Nonstandardized tidal volume}

High tidal volumes may exaggerate the size of dynamic parameters at similar preload conditions $[65,66]$. Similarly, low tidal volume may produce an inadequate change in $\mathrm{CO}$ and considerably reduce the reliability of dynamic parameters. It is accepted that such reliability can be achieved only with tidal volume $\geq 8 \mathrm{ml} / \mathrm{kg}$ [67] or $\geq 7 \mathrm{ml} / \mathrm{kg}$ [68]. Attempts at standardizing the respiratory stimulus have been suggested [15] but have not been clinically implemented.

\section{Nonstandardized airway pressure/respiratory rate}

Other instances where the airway pressure may be inappropriately elevated or decreased may complicate the interpretation of observed dynamic parameters. Similarly, a very high respiratory rate was shown to reduce the respiratory variations in SV and its derivates, whereas respiratory variations in the superior vena cava diameter were unaltered [69].

Reduced chest wall compliance (for example, severe burns or severe edema of the chest wall, circular bandages or morbid obesity) may exaggerate the hemodynamic impact of the mechanical breath and cause inappropriately high values of dynamic parameters.

Prone positioning has been shown to significantly increase PPV and SVV, although it did not alter their ability to predict fluid responsiveness [44].
Increased intra-abdominal pressure may also increase dynamic variables significantly even in the absence of fluid responsiveness [70,71].

Air-trapping and positive end-expiratory pressure may result in high values of dynamic parameters denoting a real compromise of venous return and $\mathrm{CO}$. High baseline values of dynamic variables may alert one to the possible detrimental hemodynamic effects of positive endexpiratory pressure before its application [36].

Decreased lung compliance is usually not a limitation of FHP as long as the tidal volume remains adequate. However, although some of the key clinical studies on FHP have been carried out in patients who were in respiratory failure [36], recent evidence suggests that low compliance of the respiratory system reduces the accuracy of PPV [63].

Open-chest conditions may affect the ability of respiratory variations in the arterial pressure to predict fluid responsiveness and have been the subject of some debate [22,72-75]. Since during open-chest conditions there is no inspiratory impediment to venous return, we believe that these variations are entirely due to augmentation of $\mathrm{CO}(\mathrm{dUp})$ following inspiratory squeezing of the pulmonary blood volume, that they reflect LV fluid responsiveness, and that their absence should be a cause for concern [22].

Pediatric patients are characterized by higher chest/ lung compliance and higher vascular compliance, which may account for the mixed reported results for the use of dynamic parameters in this patient population [76].

\section{Nonsinus rhythm}

Since the respiratory-induced dynamic parameters rely on the individually measured maximal and minimal SV (or its surrogates), any arrhythmias may cause significant inaccuracies. Nodal rhythm, however, may increase the size of respiratory-induced dynamic parameters by effectively decreasing preload due to the loss of the atrial kick. 


\section{A neglected dUp}

This early augmentation of LV ejection, which may become the dominant and only variation during hypervolemia and congestive heart failure $[13,14,77]$, has little volume sensitivity [22,26-28]. Since this augmentation reflects the maximal SV and hence impacts the calculation of SPV, SVV and PPV, it may reduce their accuracy in predicting fluid responsiveness, especially when their values are in mid-range. The presence of a significant dUp may explain, in part at least, the recently reported inability of PPV values between 9 and 13\% to accurately predict fluid responsiveness (gray zone) [46]. The identification of a dominant dUp, although rare, may suggest that the patient is not fluid responsive and that the mechanical breath is in fact assisting LV ejection.

\section{Right heart failure}

The inspiratory increase in right ventricular afterload may cause falsely elevated SPV, PPV or SVV in the presence of right ventricular failure, which should be suspected when a patient has large variations but does not respond to fluids.

\section{Why should functional hemodynamics be used in all surgical patients?}

Although dynamic parameters offer unique value in predicting fluid responsiveness in mechanically ventilated patients, their penetration into mainstream clinical practice has been exceedingly slow [23]. A recent survey has shown that the management of high-risk surgical patients rarely includes the actual measurement of dynamic parameters although they seem to be frequently eyeballed [10]. Similarly, very few GDT studies have included any of the dynamic parameters in their protocols $[2,3]$. The few studies that have used dynamic parameters in the context of GDT have reported nonuniform results that deserve closer examination.

Using PPV $>10 \%$ as an indication for fluid loading in a small group of high-risk surgical patients resulted in a much higher fluid administration and better outcome [39]. The very positive results of this study have to be interpreted with caution, however, as the patients of the control group seemed to receive a surprisingly small amount of fluids that may account for their reported worse outcome [39]. More recently, an SVV (>10\%)-guided approach led to the administration of more colloids, less hypotensive episodes and a lower incidence of postoperative complications [78]. In another study, where SVV $>12 \%$ was part of a GDT protocol in high-risk surgical patients, the protocol group received more colloids and less crystalloids and had a reduced length of hospital stay and a lower incidence of complications than controls [52].

Other studies have found different results. In patients undergoing major abdominal surgery, fluid management guided by a goal of SPV $<10 \%$ was also associated with slightly increased fluid administration although organ perfusion and function were not affected [79]. Similarly, the administration of a fluid bolus whenever the respiratory variation in peak aortic flow velocity was $\geq 13 \%$ did not prevent the occurrence of hypovolemia and increased the incidence of postoperative complications when a background excessive fluid restriction strategy was applied [80]. In contrast to all of the abovementioned studies, the use of the plethysmographic variation index to guide fluid administration resulted in a significantly lower amount of total volume infused intraoperatively and in lower lactate levels [81]. In summary, the current literature does not provide a robust evidence-base for the intraoperative use of FHP, one of the main reasons being the highly variable way in which the standard care group was managed during these studies.

When making individual decisions about perioperative fluid management, however, it would seem physiologically and clinically irrational to disregard the obvious insights that are offered by dynamic parameters [23-25,38]. These parameters may on one hand offer an early, and sometimes the only, sign of occult hypovolemia, and on the other may indicate when to withhold fluids since their administration may not be of benefit. This may be especially important in patients with a known impaired LV function, one-half of whom have been found to be fluid-responsive in spite of this diagnosis $[15,49]$. It is important to realize, however, that the presence of fluid-responsiveness is not an absolute indication to give fluids, and the final decision has to be supported by the apparent need for hemodynamic improvement and by the lack of associated risk. When encountering values that are within the gray zone (for example, PPV 9 to 13\%), decisions about fluid administration should be dictated by additional tools and by the risk-benefit ratio of fluid administration at that moment [46]. Last but not least, dynamic parameters may be of great help during GDT since they may indicate if and when $\mathrm{CO}$ can be further maximized by fluids, identify when the flat portion of the cardiac function curve has been reached (signifying the end of the optimization process), and prevent unnecessary fluid loading.

\section{Conclusion}

FHP offer unique information about fluid responsiveness, which may help detect fluid needs and avoid unnecessary fluid loading. Despite their limitations and confounding factors, these parameters should be used to guide fluid therapy in all surgical patients in whom their use is appropriate, as part of, or independently of, GDT strategies.

This is part of a series on Perioperative monitoring, edited by Dr Andrew Rhodes 


\section{Abbreviations}

CO, cardiac output; CVP, central venous pressure; dUp, delta up; FHP, functional hemodynamic parameters; GDT, goal-directed therapy; LV, left ventricular; PPV, pulse pressure variation; PWV, plethysmographic waveform variation; SPV, systolic pressure variation; SV, stroke volume; SW, stroke volume variation.

\section{Competing interests}

AP is a member of the medical advisory board of Pulsion Medical Systems, Munich, Germany, a consultant to BMEYE, Amsterdam, the Netherlands, and holds a patent for the Respiratory Systolic Variation Test. The remaining authors declare that they have no competing interests.

\section{Author details}

'Department of Anaesthesiology and Intensive Care Medicine, Charité Universitätsmedizin Berlin, Campus Charité Mitte and Campus Virchow Klinikum, Berlin D-10117, Germany. ${ }^{2}$ Department of Anesthesiology and Intensive Care, Sheba Medical Center, Tel Aviv University, Tel Hashomer, Tel Aviv 52621, Israel.

Published: 28 January 2013

\section{References}

1. Chappell D, Jacob M, Hofmann-Kiefer K, Conzen P, Rehm M: A rational approach to perioperative fluid management. Anesthesiology 2008 , 109:723-740.

2. Hamilton MA, Cecconi M, Rhodes A: A systematic review and meta-analysis on the use of preemptive hemodynamic intervention to improve postoperative outcomes in moderate and high-risk surgical patients. Anesth Analg 2011, 112:1392-1402.

3. Gurgel ST, Do Nascimento P: Maintaining tissue perfusion in high-risk surgical patients: a systematic review of randomized clinical trials. Anesth Analg 2011, 112:1384-1391

4. CardioQ-ODM Quick Reference Guide for the OR [http://www. deltexmedical.com/downloads/clinicaleducationguides/CQ_OR_ QRG9051_5309_3.pdf]

5. Hood JA, Wilson RJT: Pleth variability index to predict fluid responsiveness in colorectal surgery. Anesth Analg 2011, 113:1058-1063.

6. Pearse R, Dawson D, Fawcett J, Rhodes A, Grounds RM, Bennett ED: Early goal-directed therapy after major surgery reduces complications and duration of hospital stay. A randomised, controlled trial [ISRCTN38797445]. Crit Care 2005, 9:R687-R693.

7. Cecconi M, Fasano N, Langiano N, Divella M, Costa MG, Rhodes A, Rocca Della G: Goal-directed haemodynamic therapy during elective total hip arthroplasty under regional anaesthesia. Crit Care 2011, 15:R132.

8. Svensén $\mathrm{CH}, \mathrm{Olsson}$ J, Hahn RG: Intravascular fluid administration and hemodynamic performance during open abdominal surgery. Anesth Analg 2006, 103:671-676.

9. Kastrup M, Markewitz A, Spies C, Carl M, Erb J, Grosse J, Schirmer U: Current practice of hemodynamic monitoring and vasopressor and inotropic therapy in post-operative cardiac surgery patients in Germany: results from a postal survey. Acta Anaesthesio/ Scand 2007, 51:347-358.

10. Cannesson M, Pestel G, Ricks C, Hoeft A, Perel A: Hemodynamic monitoring and management in patients undergoing high risk surgery: a survey among North American and European anesthesiologists. Crit Care 2011, 15:R197.

11. Michard F, Teboul $\mathrm{J}$ : Predicting fluid responsiveness in ICU patients: a critical analysis of the evidence. Chest 2002, 121:2000-2008.

12. Marik PE, Baram M, Vahid B: Does central venous pressure predict fluid responsiveness? A systematic review of the literature and the Tale of Seven Mares. Chest 2008, 134:172-178.

13. Coriat P, Vrillon M, Perel A, Baron JF, Le Bret F, Saada M, Viars P: A comparison of systolic blood pressure variations and echocardiographic estimates of end-diastolic left ventricular size in patients after aortic surgery. Anesth Analg 1994, 78:46-53.

14. Tavernier B, Makhotine O, Lebuffe G, Dupont J, Scherpereel P: Systolic pressure variation as a guide to fluid therapy in patients with sepsisinduced hypotension. Anesthesiology 1998, 89:1313-1321.

15. Preisman S, Kogan S, Berkenstadt H, Perel A: Predicting fluid responsiveness in patients undergoing cardiac surgery: functional haemodynamic parameters including the Respiratory Systolic Variation Test and static preload indicators. Br J Anaesth 2005, 95:746-755.
16. Marik PE, Cavallazzi R, Vasu T, Hirani A: Dynamic changes in arterial waveform derived variables and fluid responsiveness in mechanically ventilated patients: a systematic review of the literature. Crit Care Med 2009, 37:2642-2647.

17. Monnet X, Teboul J-L: Volume responsiveness. Curr Opin Crit Care 2007 13:549-553.

18. Perel A: Assessing fluid responsiveness by the systolic pressure variation in mechanically ventilated patients. Anesthesiology 1998, 89:1309-1310

19. Monnet X, Letierce A, Hamzaoui O, Chemla D, Anguel N, Osman D, Richard C, Teboul J-L: Arterial pressure allows monitoring the changes in cardiac output induced by volume expansion but not by norepinephrine. Crit Care Med 2011, 39:1394-1399.

20. Antonelli M, Levy M, Andrews PJD, Chastre J, Hudson LD, Manthous C, Meduri GU, Moreno RP, Putensen C, Stewart T, Torres A: Hemodynamic monitoring in shock and implications for management. Intensive Care Med 2007 33:575-590.

21. Cecconi M, Parsons AK, Rhodes A: What is a fluid challenge? Curr Opin Crit Care 2011, 17:290-295

22. Perel $\mathrm{A}$ : The physiological basis of arterial pressure variation during positive-pressure ventilation. Réanimation 2005, 14:162-171.

23. Michard F: Changes in arterial pressure during mechanical ventilation. Anesthesiology 2005, 103:419-428.

24. Marik PE, Monnet $X$, Teboul $J$ : Hemodynamic parameters to guide fluid therapy. Annals Intensive Care 2011, 1:1

25. Cannesson M, Aboy M, Hofer CK, Rehman M: Pulse pressure variation: where are we today? J Clin Monit Comput 2010, 25:45-56.

26. Vieillard-Baron A, Chergui K, Augarde R, Prin S, Page B, Beauchet A, Jardin F: Cyclic changes in arterial pulse during respiratory support revisited by Doppler echocardiography. Am J Respir Crit Care Med 2003, 168:671-676.

27. Magder S: Further cautions for the use of ventilatory-induced changes in arterial pressures to predict volume responsiveness. Crit Care 2010, 14:197.

28. Perel A, Pizov R, Cotev S: Systolic blood pressure variation is a sensitive indicator of hypovolemia in ventilated dogs subjected to graded hemorrhage. Anesthesiology 1987, 67:498-502.

29. Mallat J, Pironkov A, Destandau M-S, Tavernier B: Systolic pressure variation (Deltadown) can guide fluid therapy during pheochromocytoma surgery. Can J Anaesth 2003, 50:998-1003.

30. Deflandre E, Bonhomme V, Hans P: Delta down compared with delta pulse pressure as an indicator of volaemia during intracranial surgery. $\mathrm{Br} J$ Anaesth 2007, 100:245-250.

31. Shamir M, Eidelman LA, Floman Y, Kaplan L, Pizov R: Pulse oximetry plethysmographic waveform during changes in blood volume. Br J Anaesth 1999, 82:178-181

32. Pizov R, Segal E, Kaplan L, Floman Y, Perel A: The use of systolic pressure variation in hemodynamic monitoring during deliberate hypotension in spine surgery. J Clin Anesth 1990, 2:96-100.

33. Michard F, Boussat S, Chemla D, Anguel N, Mercat A, Lecarpentier Y, Richard C, Pinsky MR, Teboul JL: Relation between respiratory changes in arterial pulse pressure and fluid responsiveness in septic patients with acute circulatory failure. Am J Respir Crit Care Med 2000, 162:134-138.

34. Thiele RH, Colquhoun DA, Blum FE, Durieux ME: The ability of anesthesia providers to visually estimate systolic pressure variability using the 'eyeball' technique. Anesth Analg 2012, 115:176-181.

35. Rinehart J, Islam T, Boud R, Nguyen A, Alexander B, Canales C, Cannesson M: Visual estimation of pulse pressure variation is not reliable: a randomized simulation study. J Clin Monit Comput 2012, 26:191-196.

36. Michard F, Chemla D, Richard C, Wysocki M, Pinsky MR, Lecarpentier Y, Teboul JL: Clinical use of respiratory changes in arterial pulse pressure to monitor the hemodynamic effects of PEEP. Am J Respir Crit Care Med 1999, 159:935-939.

37. Berkenstadt H, Friedman Z, Preisman S, Keidan I, Livingstone D, Perel A: Pulse pressure and stroke volume variations during severe haemorrhage in ventilated dogs. Br J Anaesth 2005, 94:721-726.

38. Michard F, Lopes MR, Auler JOC: Pulse pressure variation: beyond the fluid management of patients with shock. Crit Care 2007, 11:131.

39. Lopes MR, Oliveira MA, Pereira VOS, Lemos IPB, Auler JOC, Michard F: Goaldirected fluid management based on pulse pressure variation monitoring during high-risk surgery: a pilot randomized controlled trial. Crit Care 2007, 11:R100.

40. Gouvea G, Diaz R, Auler L, Toledo R, Martinho JM: Evaluation of the pulse pressure variation index as a predictor of fluid responsiveness during orthotopic liver transplantation. Br J Anaesth 2009, 103:238-243. 
41. Kramer A, Zygun D, Hawes H, Easton P, Ferland A: Pulse pressure variation predicts fluid responsiveness following coronary artery bypass surgery. Chest 2004, 126:1563-1568.

42. Belloni L, Pisano A, Natale A, Piccirillo MR, Piazza L, Ismeno G, De Martino G: Assessment of fluid-responsiveness parameters for off-pump coronary artery bypass surgery: a comparison among LiDCO, transesophageal echochardiography, and pulmonary artery catheter. J Cardiothorac VasC Anesth 2008, 22:243-248.

43. Auler JO, Jr, Galas F, Hajjar L, Santos L, Carvalho T, Michard F: Online monitoring of pulse pressure variation to guide fluid therapy after cardiac surgery. Anesth Analg 2008, 106:1201-1206.

44. Biais M, Bernard O, Ha JC, Degryse C, Sztark F: Abilities of pulse pressure variations and stroke volume variations to predict fluid responsiveness in prone position during scoliosis surgery. Br J Anaesth 2010, 104:407-413.

45. Murugan R, Venkataraman R, Wahed AS, Elder M, Carter M, Madden NJ, Kellum A: Preload responsiveness is associated with increased interleukin- 6 and lower organ yield from brain-dead donors. Crit Care Med 2009, 37:2387-2393.

46. Cannesson M, Le Manach Y, Hofer CK, Goarin JP, Lehot J-J, Vallet B, Tavernier B. Assessing the diagnostic accuracy of pulse pressure variations for the prediction of fluid responsiveness: a'gray zone' approach. Anesthesiology 2011, 115:231-241

47. Berkenstadt H, Margalit N, Hadani M, Friedman Z, Segal E, Villa Y, Perel A: Stroke volume variation as a predictor of fluid responsiveness in patients undergoing brain surgery. Anesth Analg 2001, 92:984-989.

48. Reuter DA, Felbinger TW, Schmidt C, Kilger E, Goedje O, Lamm P, Goetz AE: Stroke volume variations for assessment of cardiac responsiveness to volume loading in mechanically ventilated patients after cardiac surgery. Intensive Care Med 2002, 28:392-398.

49. Reuter DA, Kirchner A, Felbinger TW, Weis FC, Kilger E, Lamm P, Goetz AE: Usefulness of left ventricular stroke volume variation to assess fluid responsiveness in patients with reduced cardiac function. Crit Care Med 2003, 31:1399-1404.

50. Hofer CK, Senn A, Weibel L, Zollinger A: Assessment of stroke volume variation for prediction of fluid responsiveness using the modified FloTrac and PiCCOplus system. Crit Care 2008, 12:R82.

51. Cannesson M, Musard H, Desebbe O, Boucau C, Simon R, Hénaine R, Lehot J-J: The ability of stroke volume variations obtained with Vigileo/FloTrac system to monitor fluid responsiveness in mechanically ventilated patients. Anesth Analg 2009, 108:513-517

52. Mayer J, Boldt J, Mengistu AM, Röhm KD, Suttner S: Goal-directed intraoperative therapy based on autocalibrated arterial pressure waveform analysis reduces hospital stay in high-risk surgical patients: a randomized, controlled trial. Crit Care 2010, 14:R18.

53. Kobayashi M, Koh M, Irinoda T, Meguro E, Hayakawa Y, Takagane A: Stroke volume variation as a predictor of intravascular volume depression and possible hypotension during the early postoperative period after esophagectomy. Ann Surg Oncol 2009, 16:1371-1377.

54. Biais M, Nouette-Gaulain K, Cottenceau V, Revel P, Sztark F: Uncalibrated pulse contour-derived stroke volume variation predicts fluid responsiveness in mechanically ventilated patients undergoing liver transplantation. $\mathrm{Br} J$ Anaesth 2008, 101:761-768.

55. Perel A: Automated assessment of fluid responsiveness in mechanically ventilated patients. Anesth Analg 2008, 106:1031-1033.

56. Maguire S, Rinehart J, Vakharia S, Cannesson M: Respiratory variation in pulse pressure and plethysmographic waveforms. Anesth Analg 2011, 112:94-96.

57. Pizov R, Eden A, Bystritski D, Kalina E, Tamir A, Gelman S: Arterial and plethysmographic waveform analysis in anesthetized patients with hypovolemia. Anesthesiology 2010, 113:83-91.

58. Cannesson M, Desebbe O, Rosamel P, Delannoy B, Robin J, Bastien O, Lehot J Pleth variability index to monitor the respiratory variations in the pulse oximeter plethysmographic waveform amplitude and predict fluid responsiveness in the operating theatre. Br J Anaesth 2008, 101:200-206.

59. Solus-Biguenet H, Fleyfel M, Tavernier B, Kipnis E, Onimus J, Robin E, Lebuffe G, Decoene C, Pruvot FR, Vallet B: Non-invasive prediction of fluid responsiveness during major hepatic surgery. Br J Anaesth 2006, 97:808-816.

60. Wyffels PAH, Durnez P-J, Helderweirt J, Stockman WMA, De Kegel D: Ventilation-induced plethysmographic variations predict fluid responsiveness in ventilated postoperative cardiac surgery patients. Anesth Analg 2007, 105:448-452

61. Cavallaro F, Sandroni C, Marano C, La Torre G, Mannocci A, De Waure C, Bello G, Maviglia R, Antonelli M: Diagnostic accuracy of passive leg raising for prediction of fluid responsiveness in adults: systematic review and metaanalysis of clinical studies. Intensive Care Med 2010, 36:1475-1483.

62. Monnet X, Osman D, Ridel C, Lamia B, Richard C, Teboul J-L: Predicting volume responsiveness by using the end-expiratory occlusion in mechanically ventilated intensive care unit patients. Crit Care Med 2009, 37:951-956.

63. Monnet X, Bleibtreu A, Ferré A, Dres M, Gharbi R, Richard C, Teboul J-L: Passive leg-raising and end-expiratory occlusion tests perform better than pulse pressure variation in patients with low respiratory system compliance. Crit Care Med 2012, 40:152-157.

64. Perel A: The value of functional hemodynamic parameters in hemodynamic monitoring of ventilated patients. Anaesthesist 2003, 52:1003-1004.

65. Szold A, Pizov R, Segal E, Perel A: The effect of tidal volume and intravascular volume state on systolic pressure variation in ventilated dogs. Intensive Care Med 1989, 15:368-371.

66. Reuter DA, Bayerlein J, Goepfert MSG, Weis FC, Kilger E, Lamm P, Goetz AE: Influence of tidal volume on left ventricular stroke volume variation measured by pulse contour analysis in mechanically ventilated patients. Intensive Care Med 2003, 29:476-480.

67. Backer D, Heenen S, Piagnerelli M, Koch M, Vincent J-L: Pulse pressure variations to predict fluid responsiveness: influence of tidal volume. Intensive Care Med 2005, 31:517-523.

68. Lansdorp B, Lemson J, van Putten MJAM, de Keijzer A, van der Hoeven JG, Pickkers P: Dynamic indices do not predict volume responsiveness in routine clinical practice. Br J Anaesth 2012, 108:395-401.

69. De Backer D, Taccone FS, Holsten R, Ibrahimi F, Vincent JL: Influence of respiratory rate on stroke volume variation in mechanically ventilated patients. Anesthesiology 2009, 110:1092-1097

70. Malbrain ML, de Laet I: Functional hemodynamics and increased intraabdominal pressure: same thresholds for different conditions ...? Crit Care Med 2009, 37:781-783.

71. Mahjoub Y, Touzeau J, Airapetian N, Lorne E, Hijazi M, Zogheib E, Tinturier F, Slama M, Dupont $\mathrm{H}$ : The passive leg-raising maneuver cannot accurately predict fluid responsiveness in patients with intra-abdominal hypertension. Crit Care Med 2010, 38:1824-1829.

72. Sander M, Spies CD, Berger K, Grubitzsch H, Foer A, Krämer M, Carl M, Heymann Von C: Prediction of volume response under open-chest conditions during coronary artery bypass surgery. Crit Care 2007, 11:R121

73. Teboul J-L: Meaning of pulse pressure variation during cardiac surgery: everything is open. Crit Care Med 2009, 37:757-758

74. Wyffels PAH, Sergeant P, Wouters PF: The value of pulse pressure and stroke volume variation as predictors of fluid responsiveness during open chest surgery. Anaesthesia 2010, 65:704-709.

75. De Waal EE, Rex S, Kruitwagen CL, Kalkman CJ, Buhre WF: Dynamic preload indicators fail to predict fluid responsiveness in open-chest conditions. Crit Care Med 2009, 37:510-515.

76. Chung $E$, Cannesson M: Using non invasive dynamic parameters of fluid responsiveness in children: there is still much to learn. J Clin Monit Comput 2012, 26:153-155

77. Pizov $R$, Ya'ari Y, Perel $A$ : The arterial pressure waveform during acute ventricular failure and synchronized external chest compression. Anesth Analg 1989, 68:150-156.

78. Benes J, Chytra I, Altmann P, Hluchy M, Kasal E, Svitak R, Pradl R, Stepan M: Intraoperative fluid optimization using stroke volume variation in high risk surgical patients: results of prospective randomized study. Crit Care 2010, 14:R118.

79. Buettner M, Schummer W, Huettemann E, Schenke S, van Hout N, Sakka SG: Influence of systolic-pressure-variation-guided intraoperative fluid management on organ function and oxygen transport. Br J Anaesth 2008, 101:194-199.

80. Futier E, Constantin J-M, Petit A, Chanques G, Kwiatkowski F, Flamein R, Slim K, Sapin $V$, Jaber S, Bazin J-E: Conservative vs restrictive individualized goaldirected fluid replacement strategy in major abdominal surgery: a prospective randomized trial. Arch Surg 2010, 145:1193-1200.

81. Forget $P$, Lois F, de Kock M: Goal-directed fluid management based on the pulse oximeter-derived pleth variability index reduces lactate levels and improves fluid management. Anesth Analg 2010, 111:910-914.

doi:10.1186/cc11448

Cite this article as: Perel A, et al:: Bench-to-bedside review: Functional hemodynamics during surgery - should it be used for all high-risk cases? Critical Care 2013, 17:203. 